\title{
What Drives the US and Peruvian HIV Epidemics in Men Who Have Sex with Men (MSM)?
}

\author{
Steven M. Goodreau ${ }^{1 *}$, Nicole B. Carnegie ${ }^{2}$, Eric Vittinghoff ${ }^{3}$, Javier R. Lama ${ }^{4}$, Jorge Sanchez ${ }^{5}$, \\ Beatriz Grinsztejn ${ }^{6}$, Beryl A. Koblin 7 , Kenneth H. Mayer ${ }^{8}$, Susan P. Buchbinder ${ }^{9}$
}

1 Department of Anthropology, University of Washington, Seattle, Washington, United States of America, 2 Department of Biostatistics, Harvard School of Public Health, Boston, Massachusetts, United States of America, 3 Department of Epidemiology and Biostatistics, University of California San Francisco, San Francisco, California, United States of America, 4 Asociación Civil Impacta Salud y Educación, Lima, Peru, 5 Asociación Civil Impacta Salud y Educación, Lima, Peru, 6 Instituto de Pesquisa Clinica Evandro Chagas, Fundação Oswaldo Cruz, Rio de Janeiro, Brazil, 7 Laboratory of Infectious Disease Prevention, New York Blood Center, New York City, New York, United States of America, 8 The Fenway Institute, Fenway Health, Boston, Massachusetts, United States of America, 9 Bridge HIV, San Francisco Department of Public Health, San Francisco, California, United States of America

\begin{abstract}
In this work, we estimate the proportions of transmissions occurring in main vs. casual partnerships, and by the sexual role, infection stage, and testing and treatment history of the infected partner, for men who have sex with men (MSM) in the US and Peru. We use dynamic, stochastic models based in exponential random graph models (ERGMs), obtaining inputs from multiple large-scale MSM surveys. Parallel main partnership and casual sexual networks are simulated. Each man is characterized by age, race, circumcision status, sexual role behavior, and propensity for unprotected anal intercourse (UAI); his history is modeled from entry into the adult population, with potential transitions including HIV infection, detection, treatment, AIDS diagnosis, and death. We implemented two model variants differing in assumptions about acute infectiousness, and assessed sensitivity to other key inputs. Our two models suggested that only $4-5 \%$ (Model 1 ) or $22-29 \%$ (Model 2) of HIV transmission results from contacts with acute-stage partners; the plurality ( $80-81 \%$ and $49 \%$, respectively) stem from chronic-stage partners and the remainder (14-16\% and $27-35 \%$, respectively) from AIDS-stage partners. Similar proportions of infections stem from partners whose infection is undiagnosed (24-31\%), diagnosed but untreated (36-46\%), and currently being treated (30-36\%). Roughly one-third of infections (32-39\%) occur within main partnerships. Results by country were qualitatively similar, despite key behavioral differences; one exception was that transmission from the receptive to insertive partner appears more important in Peru (34\%) than the US (21\%). The broad balance in transmission contexts suggests that education about risk, careful assessment, pre-exposure prophylaxis, more frequent testing, earlier treatment, and risk-reduction, disclosure, and adherence counseling may all contribute substantially to reducing the HIV incidence among MSM in the US and Peru.
\end{abstract}

Citation: Goodreau SM, Carnegie NB, Vittinghoff E, Lama JR, Sanchez J, et al. (2012) What Drives the US and Peruvian HIV Epidemics in Men Who Have Sex with Men (MSM)? PLoS ONE 7(11): e50522. doi:10.1371/journal.pone.0050522

Editor: Patrick S. Sullivan, Rollins School of Public Health, Emory University, United States of America

Received July 28, 2012; Accepted October 23, 2012; Published November 29, 2012

Copyright: $\odot 2012$ Goodreau et al. This is an open-access article distributed under the terms of the Creative Commons Attribution License, which permits unrestricted use, distribution, and reproduction in any medium, provided the original author and source are credited.

Funding: The study was supported by a grant (R01-Al083060) from the National Institute of Allergy and Infectious Disease (NIAID) and the National Institute of Mental Health (NIMH), United States National Institutes of Health. The funders had no role in study design, data collection and analysis, decision to publish, or preparation of the manuscript.

Competing Interests: The authors have declared that no competing interests exist.

*E-mail: goodreau@uw.edu

\section{Introduction}

Three decades on, the HIV epidemic in the United States and other highly developed nations remains concentrated among men who have sex with men (MSM), with over half of new infections occurring in this community [1]. The same is true throughout Latin America [2]. In the US, HIV incidence is rising among young MSM, especially Blacks [3]. Incidence trends for Latin American MSM are less well characterized, although prevalence remains high in this community throughout the region [4].

Recent advances in pre-exposure prophylaxis [5], testing [6], antiretroviral treatment-as-prevention [7] and circumcision $[8,9,10]$ have raised hopes for HIV prevention, including among MSM. While none alone is likely to stop the epidemic, larger reductions in incidence may be achieved by prevention packages, tailored to individual risks and preferences, that combine some or all of these new interventions with new and existing counseling approaches that support adherence, more frequent HIV testing, and continued sexual risk reduction.

Optimizing prevention packages will require a better understanding of HIV transmission in these populations. Recent work has estimated the proportion of new infections among developedworld MSM that occur within main partnerships [11,12,13,14], during acute infection $[15,16,17,18,19,20,21,22,23,24]$, and by the sexual role [13], testing history, and treatment status of the infected partner $[22,25]$. These studies use methods ranging from cross-sectional modeling to phylogenetics, start from varying assumptions, and typically address only one or two of these questions. Similar work on MSM transmission dynamics in developing world settings is lacking.

In this paper we jointly estimate these key epidemiological measures among urban MSM populations in the US and Peru. Both countries have epidemics concentrated among MSM [2], and, despite population differences in socioeconomic status, 
health care access, and sexual behaviors, HIV prevalence among urban MSM in each country appears to be roughly similar [26,27]. This work represents the baseline modeling results for the Prevention Umbrella for MSM in the Americas (PUMA) project, which aims to identify more effective ways of combining and targeting HIV interventions for MSM throughout the Western Hemisphere.

\section{Methods}

\section{Overview}

We use dynamic, stochastic, network models, one for the US and one for Peru, parameterized using multiple large-scale behavioral and demographic surveys of MSM, including NHBS [26,28], Peru's Sentinel Surveillance [29], HPTN-036 [30], and the baseline surveys of HPTN-039 [31] and Explore [32,33]. All are focused on large urban centers, and most have some definition of high risk for their inclusion criteria, so our models reflect these populations. The models simulate parallel networks for main partnerships and casual sexual contacts, both implemented using exponential random graph models (ERGMs) in the $\mathrm{R}$ package suite statnet [34], with some extensions. The main/casual distinction parallels that from source studies, which generally define a main partnership in terms of emotional commitment. Additional model components are also programmed in R. Model features and inputs were determined in consultation with a team of HIV researchers and community advisory board members. Unlike compartmental models, network models are not easily presented through flow diagrams; instead, we provide an analogous summary of model structure in Table 1. Additional detail, including parameter values, is provided in the Supporting Information.

\section{Overall Population}

Our models work by simulating the day-by-day history of a population of MSM, beginning with 10,000 men. Individuals vary according to fixed characteristics including race, circum- cision status, sexual role, and propensity for UAI in casual sexual contact; sexual role is modeled as varying continuously from exclusively insertive to exclusively receptive. Men enter the population at age 18 and are followed until age 65 or death, whichever comes first. The assumed entry rate results in slow population growth in the presence of a stable HIV epidemic. Non-AIDS-related mortality rates are based on published life tables; in men who never receive treatment, disease progression to AIDS occurs 10 years after HIV infection, and death from AIDS occurs 2 years later; progression is slower and survival greater for men on ART, and varies by magnitude of adherence and viral suppression (see below). Models were begun with $19 \%$ prevalence [26], run through a burn-in period to eliminate the transient effects of seeding the infection arbitrarily, and then compared to observed HIV incidence and prevalence in these populations. We discuss the burn-in in more depth in the online technical supplement, and its relationship to our outcomes. Each scenario is modeled 10 times, for 25 years.

\section{Main Partnerships}

Day by day, we simulate the formation and subsequent dissolution of main partnerships, the occurrence of unprotected anal intercourse (UAI) within them, and HIV transmission if one partner is infected. Under the ERGM, main partnership formation reflects each potential partner's sexual role, race, age, and current partnership status. Partnerships between men with incompatible sexual roles (two strictly insertive or receptive men) are disallowed. After formation, the daily risk of main partnership dissolution is assumed constant, so partnership duration follows a geometric distribution. If either partner is infected, daily UAI probability varies by whether the infection has been diagnosed and disclosed. UAI rates are further reduced once either man develops clinical AIDS. Sexual role may vary across UAI episodes, according to the sexual roles of both partners.

Table 1. Model features.

\begin{tabular}{|c|c|c|}
\hline $\begin{array}{l}\text { Agent attributes. Each man (agent) in the } \\
\text { model possesses the following attributes: }\end{array}$ & $\begin{array}{l}\text { Network models. The probabilities governing } \\
\text { relations between pairs: }\end{array}$ & $\begin{array}{l}\text { Transitions. The following changes can } \\
\text { happen to men: }\end{array}$ \\
\hline Age & Main partnerships evolve as a function of: & Entrance into population \\
\hline Race (US only) & Age of men & Aging \\
\hline Circumcision status & Race of men (US only) & Infection \\
\hline Sexual role preference & \# of partnerships men are already in & Change in viral load \\
\hline Propensity for casual UAI & UAI occurs w/in main partnerships as function of: & Progression through disease stages \\
\hline Infection status & Disclosure and diagnosis status of men & Testing \\
\hline Diagnosis status & Disease stage of men & Treatment initiation \\
\hline Viral load (for HIV+ men) & Men's sexual roles & Death from AIDS \\
\hline Stage of infection (for HIV+ men) & Casual UAI occurs as a function of: & Death from other causes \\
\hline Treatment status (for HIV+ men) & Age of men & Sexual retirement \\
\hline Treatment adherence and suppression & Race of men (US only) & \\
\hline Testing propensity & Men's propensity for casual UAI & \\
\hline Duration since sexual debut & Men's disclosure and diagnosis status & \\
\hline Duration since last negative test & Disease stage of men & \\
\hline Duration since infection & Men's sexual roles & \\
\hline Duration since positive diagnosis & & \\
\hline
\end{tabular}




\section{Casual Sexual Contacts}

In parallel, we simulate UAI in casual contacts and resulting HIV transmission. Specific contacts reflect the sexual role, age, race, and HIV serostatus of each partner, as well as his propensity for UAI in casual contacts. As with main partnerships, contacts between men with incompatible sexual roles are disallowed, and only UAI risk is considered.

\section{HIV Transmission}

When a UAI contact occurs between serodiscordant partners, per-contact HIV transmission risk is made to depend on the role of the uninfected partner, and if he is insertive, whether he is circumcised. In the US model, circumcision prevalence varies by race, and declines over time, while for Peru prevalence is constant. Per-contact transmission risk is also made to depend on the time since infection of the infected partner as well as his treatment status, mediated by viral load, as described below.

Transmission probabilities are not as well documented for MSM as for heterosexuals; the one serodiscordant couple study of transmission by time since infection focused on heterosexual transmission [35], as have all three studies of circumcision $[8,9,10]$. Our initial approach (Model 1) focused exclusively on viral load differences as the source of acute hyperinfectiousness. However, new research published during the course of this project suggests that acute infection viral strains may be more infectious even after accounting for viral load [36,37]. We thus implemented a second variant (Model 2) that considered these additional potential sources. We explore both models for both countries.

In Model 1, we based infection rates on viral load and percontact risk in the absence of treatment, modeled with parametric curves $[38,39,40]$. These estimates did not take into account stage of infection independent of viral load. Viral load rises and falls over 40 days post-infection; chronic infection lasts for a subsequent ten years in the absence of treatment, followed by two years of rising viral load during late-stage infection; then death. Per-contact risk ratios by sexual role and circumcision status of an uninfected insertive partner were determined by estimates for anal [41] and vaginal sex [8], respectively.

In Model 2, we used infection stage and per-contact risk in the absence of treatment, without explicit consideration of viral load. The acute stage is assumed to last two months, with infectiousness rising in the first month and falling in the second; subsequent changes in per-contact risk follow the patterns from Model 1, except for the slope of infectiousness during late-stage. We derived risk ratios for the average risk in each stage from estimates of relative per-contact risk by stage for UAI among heterosexuals [35]; infectiousness slopes during acute and late stages were fit to yield these average stage-specific relative risks. Risk ratios by role and circumcision status were carried over from Model 1. Given uncertainty in absolute risk in this framework, per-contact risks were calibrated to yield HIV prevalence for the stable epidemic equal to Model 1.

The infectiousness curve implied by each model in the absence of treatment is summarized by Figure la (acute stage) and Figure $1 \mathrm{~b}$ (AIDS stage).

\section{Testing, Treatment, and Infectiousness}

We simulate HIV testing, and, after an infection has been diagnosed, when treatment begins. In both models, infectiousness on treatment is mediated by viral load.

Men become eligible for treatment initiation when their expected CD4+ count, modeled as a function of time since infection [42], drops below country-specific thresholds, which are also race-specific for the US. Thresholds are determined by observed CD4+ counts at initiation, and are driven by a variety of factors such as testing frequencies and access to health care. Explicitly modeling a wide range of patterns of treatment initiation, levels of adherence, cessation, and potential reinitiation was beyond the scope of the model, since the data needed to do so were limited (particularly in terms of survival for men with arbitrarily complex treatment histories). Instead, we reduce this complexity down to three representative trajectories: a proportion of randomly selected men in each country never initiate treatment; among those who do, some achieve partial and some full viral suppression, depending on adherence. Parameters are set to achieve the prevalence of three states in cross-sectional data: the proportion of men who are not on treatment; on treatment and partially suppressed; and on treatment and fully suppressed. Rates of progression to AIDS and death, as well as infectiousness, vary across these groups. In particular, per-contact risks are motivated by published estimates of mean viral load [43] for each group, in combination with the relationship of viral load to infectiousness from Model 1. With disease progression, viral load and thus infectiousness increase again until death [40]. Results suggest a $93 \%$ reduction for full suppression as compared to untreated men, qualitatively similar to the $96 \%$ recently reported [7].

\section{Sensitivity Analyses}

Models 1 and 2 vary in their treatment of infectiousness early in infection. To obtain estimates of uncertainty due to key model inputs, we conducted sensitivity analyses varying three particularly uncertain and/or influential inputs: duration of main partnerships; relative frequency of UAI in main partnerships and casual contacts; and HIV testing frequency.

\section{Results}

\section{Prevalence and Incidence}

In Model 1, prevalence at steady state across repeated simulations averages 28\% for the US and 29\% for Peru, and mean annual incidence is $1.8 \%$ and $1.9 \%$, respectively. The US prevalence estimate is slightly higher than the 25\% found in most cities in the first MSM round of NHBS [44], an expected pattern given that most source datasets excluded MSM with no HIV risk. For Peru, there is less clear documentation of recent incidence and prevalence. Reports of HIV prevalence for Lima in the early 2000s were just above $20 \%$, and increasing by $6 \%$ year over year [27]; if that trend has continued, prevalence should be $\sim 30 \%$ in recent years. Other Peruvian cities have lower prevalence, but our model mainly reflects inputs for Lima, home to $73 \%$ of the country's diagnoses [45]; source data here also exclude men with no HIV risk.

\section{Infection Stage, Diagnosis and Treatment}

Model 1- in which infectiousness is mediated by viral loadpredicts only a small role for acute infection $5 \%$ in the US and $4 \%$ in Peru) and a dominant role for chronic infection $(81 \%$ and $80 \%$, respectively; Figure 2). In contrast, Model 2-which uses a stage-based model for viral load in the absence of treatment- estimates a more equal balance, but still a plurality for chronic $(29 \%, 44 \%, 27 \%$ for acute/chronic/late in the US; $22 \%, 43 \%, 35 \%$ in Peru). The distribution of transmissions by detection and treatment also varies by model; nevertheless, in all cases transmissions are roughly balanced across the undiagnosed, untreated, and treated groups. 
Model 1

a) Acute stage

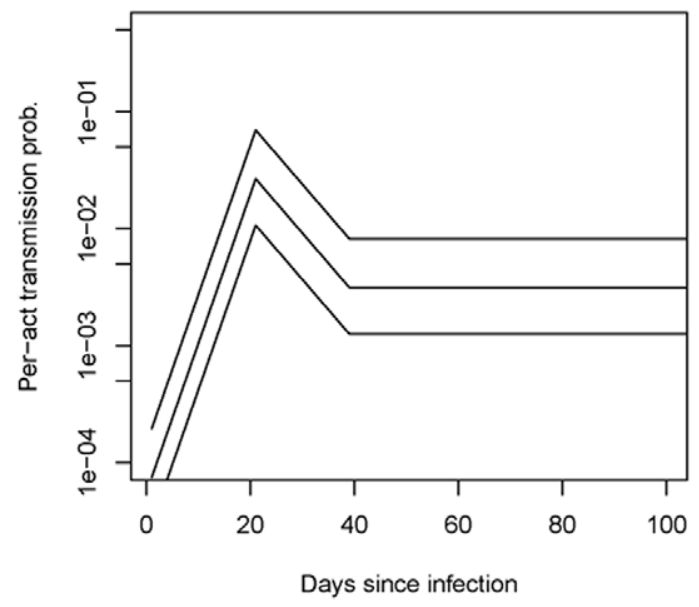

Model 1

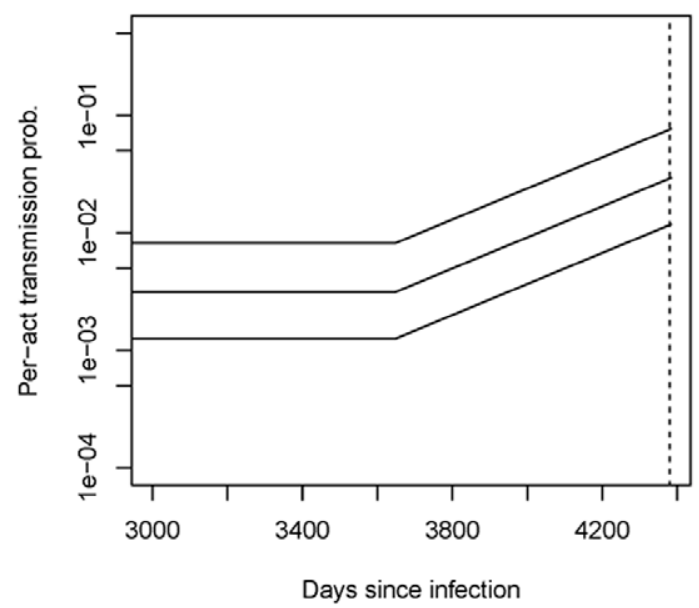

Model 2

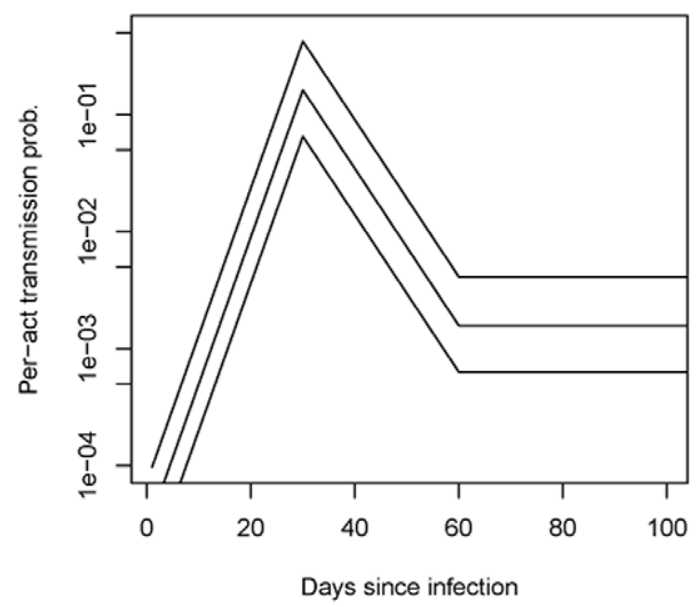

Model 2

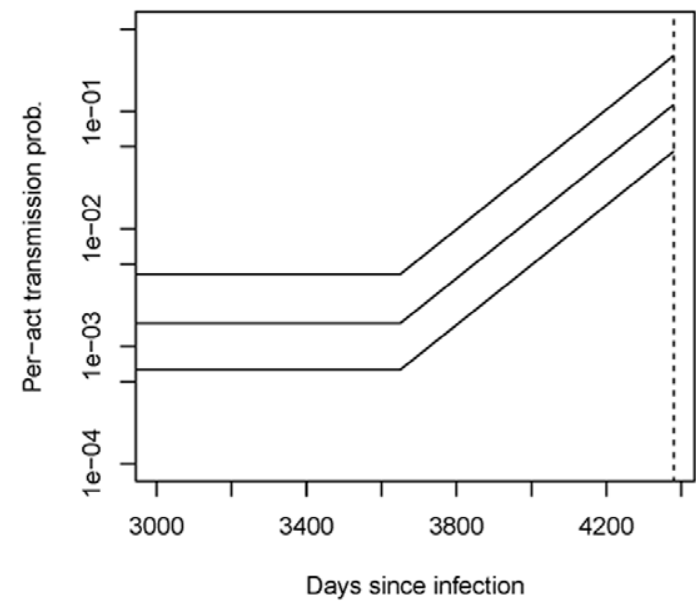

Figure 1. Per-act infectivity by time since infection, during acute stage (Figure 1a) and AIDS state (Figure 1b). For more information on the derivation, see the online technical supplement.

doi:10.1371/journal.pone.0050522.g001

\section{Proportion of HIV Transmission Occurring within Main Partnerships}

Model 1 consistently estimates that UAI within main partnerships is the source of $\sim 39 \%$ of infections in the US and $\sim 35 \%$ in Peru, despite constituting $52 \%$ and $48 \%$ of UAI acts, respectively. Results for Model 2 are slightly lower.

\section{Sexual Role}

Transmissions by sexual role showed the greatest international difference. Model 1 estimated that in the US, only $21 \%$ of transmission was from the receptive to the insertive partner, compared to $34 \%$ for Peru; Model 2 is qualitatively similar. This can be partly attributed to the lower prevalence of circumcisionassumed to lower UIAI risk by $60 \%$-in Peru, in combination with greater role exclusivity; in the latter context, transmission to moreor-less exclusively insertive men must play a bigger role in sustaining chains of infection.

\section{Variation in Outcomes}

Our metrics on transmission types varied little across runs within the same scenario, never ranging over more than 2 percentage points. This is due to both our large population size and to the long time period ( 25 years) over which we modeled them. Variation in metrics was of course higher over the short term, and this short-term variation can be of interest. Figure 3 shows the distribution for each outcome metric when measured for each of the 25 years separately, for the US Model 1. Analogous plots for the other country/model combinations are in the online technical supplement. Each shows a qualitatively similar pattern: for our population, these metrics see most of their variation lie with a roughly ten percentage-point range. It is important to remember that the magnitude of this range is still a function of our population size ( 10,000, with roughly 150 new infections per year). Smaller populations would see more variation; those that are larger, with sexual partnerships forming throughout the population, would see less. 


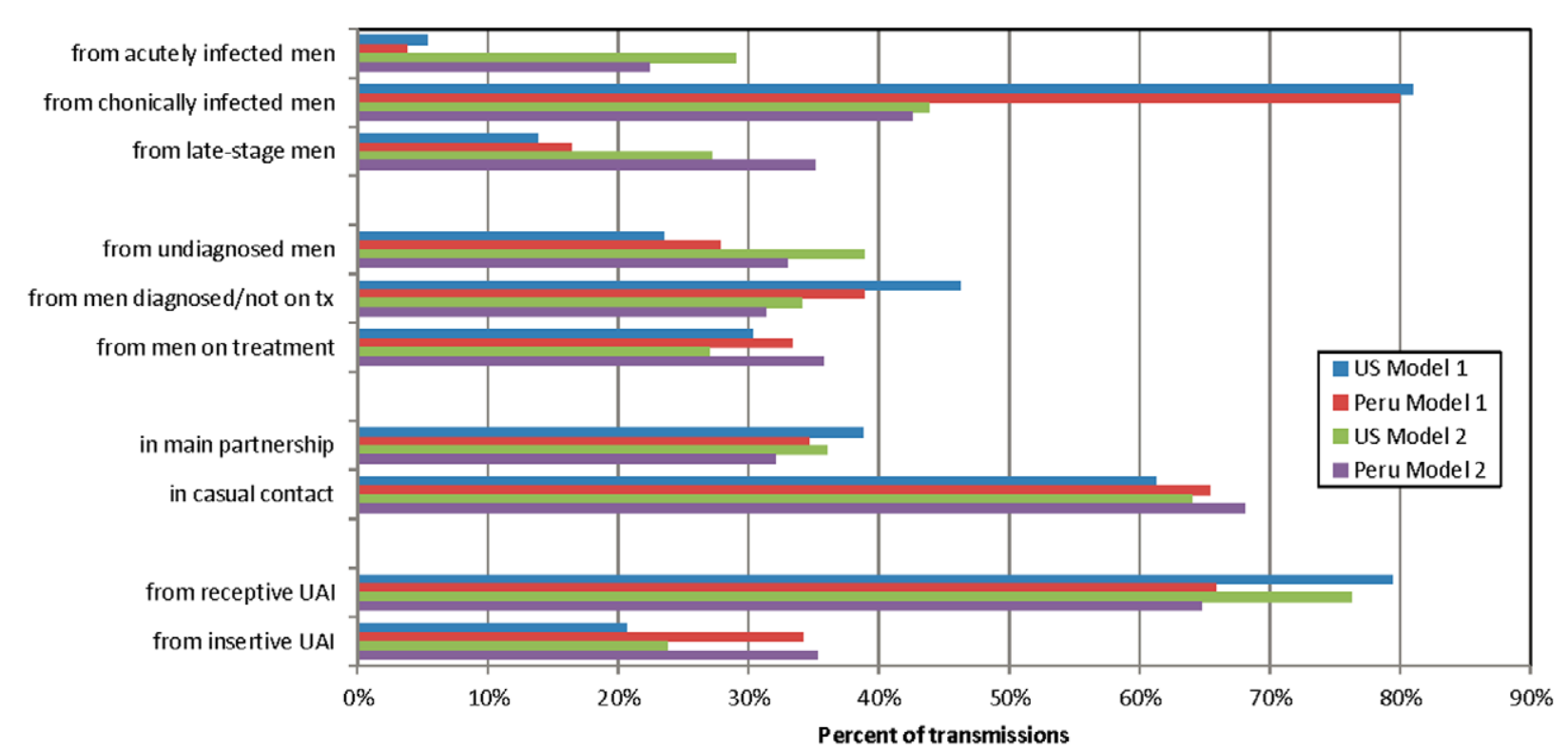

Figure 2. Distribution of transmission events by key variables. Bars represent the means across ten stochastic simulations over 25 years. The variance of these estimates across runs was less than 2 percentage points for all measures, so only means are shown. For year-on-year variation, see the Figure 3 and the online technical supplement.

doi:10.1371/journal.pone.0050522.g002

\section{Sensitivity Analyses}

Analyses were conducted to assess sensitivity to three particularly uncertain model inputs. We present these for Model 1 in Figure 4. Average main partnership duration is hard to estimate, with a strongly right-skewed distribution, censoring, and recall bias. We varied this input, holding the prevalence of main partnerships constant (Figure 4a). Results were only mildly sensitive to partnership length.

Given the divergence between our estimates of the proportion of transmissions occurring in main partnerships and other published estimates, we explored the impact of different assumptions about the relative frequency of UAI in main partnerships versus casual contacts (Figure 4b). Specifically, we increased the frequency in main partnerships by $10-40 \%$, simultaneously reducing its frequency in casual contacts by the same percentage. As expected, the proportion of transmissions occurring in main partnerships correlates positively with the relative frequency of UAI in these partnerships. However, it remains much lower than the proportion of all UAI contacts occurring in these partnerships, reflecting the fact that transmission can only occur once in any partnership. Moreover, the estimated proportion of main-partner transmissions never exceeds $50 \%$, even with very large increases in the relative UAI frequency; this reflects the need for casual contacts to sustain chains of infection.

Data on the proportion of men reporting testing in the last year, used in our models, generally suggest higher testing frequency than estimates based on self-reported infection status among men testing positive $[26,44,46]$. Even with testing at half our estimated frequency (Figure 4c), men with diagnosed infection generate a majority of transmissions. At lower testing rates, this proportion necessarily drops, but reaches a lower bound; in addition, HIV prevalence in these models increases to less realistic levels.

\section{Discussion}

Our models are the first to comprehensively and consistently address several questions about HIV transmission, including the proportions of transmissions occurring during acute as well as undiagnosed, untreated, and partially suppressed infection, within main partnerships, and to insertive partners in anal intercourse. Our results help clarify these questions for MSM in sites on two continents, with implications for prioritizing prevention efforts and designing combination prevention interventions, including the PUMA package currently in development.

Although variation in infectiousness soon after HIV acquisition is not well understood for MSM, leading us to develop two variants of our models, nonetheless both suggested that a considerably smaller percentage of transmissions occur during acute infection than some recent estimates based on phylogenetic clusters $[15,18,24,47]$. Newer work has critiqued the statistical methods used in some of those papers, and developed their own estimates based on similar data types [16,17]; these, as well as other modeling estimates [19,20,23], lie closer to our more conservative estimate of 4-5\%. Our less conservative estimates of $22 \%$ in Peru and 29\% in the US are more similar to one modeling estimate for Australia [22]. Recent work [36,37] suggesting that additional factors beyond just viral load induce high transmissibility during acute infection would argue for the less conservative estimates, although further clarification on acute infectiousness for MSM is urgently needed. The lower estimate for Peru may reflect lower circumcision prevalence, later treatment initiation, and less suppression due to lower adherence among the treated, all of which make post-acute transmission more likely. However, all of our estimates suggest that strategies to increase testing for men regardless of stage of infection may be more fruitful than some phylogenetic work seems to imply, and that efforts focused narrowly on the more difficult task of identifying acute-stage men are not as crucial. 


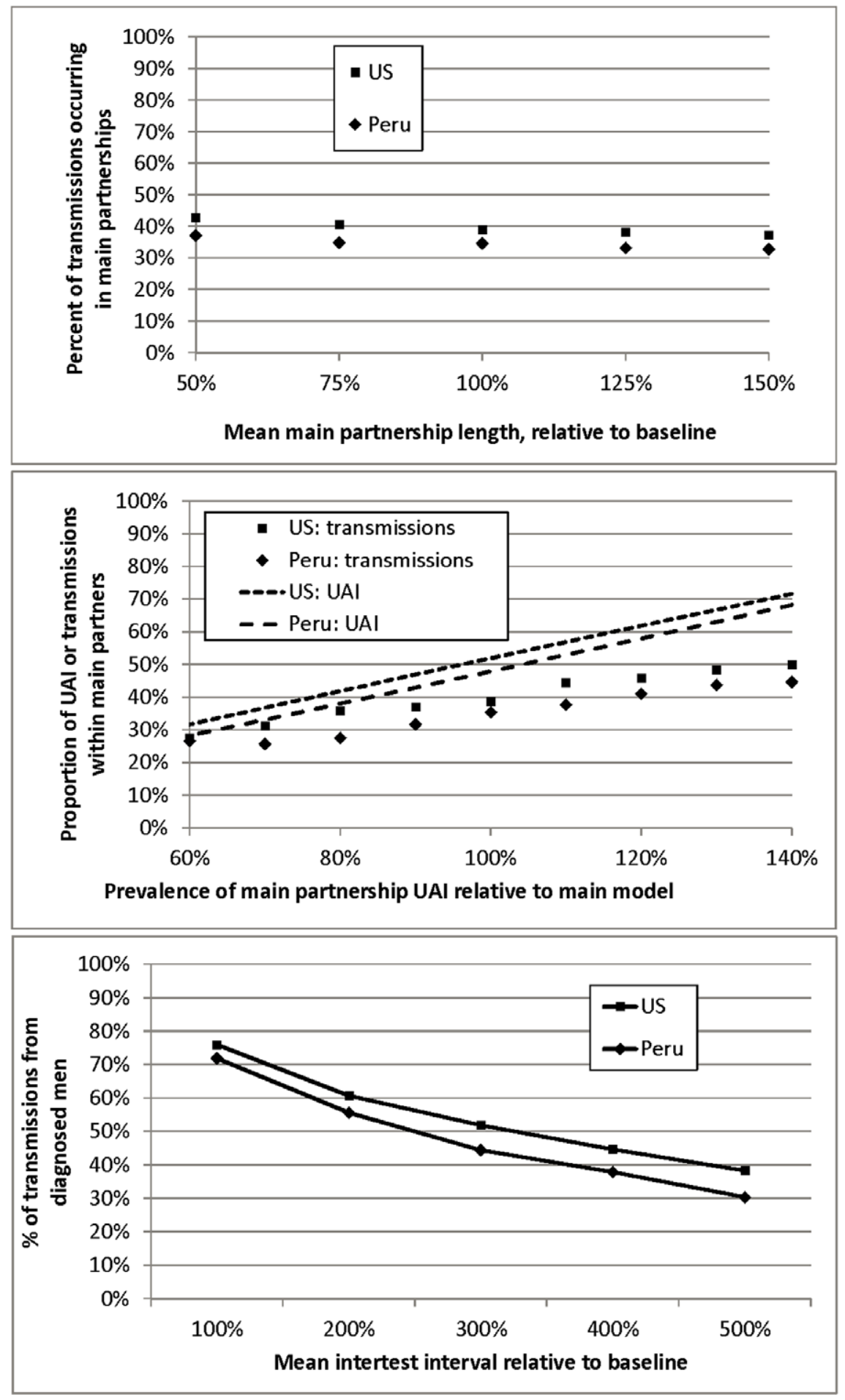

Figure 3. Range of variation from year to year for outcome metrics. Each boxplot covers the values for a given outcome metric measured for each of the 25 years in a single run. We show US Model 1 here as demonstration; comparable plots for the other three country/model combinations are in the online technical supplement.

doi:10.1371/journal.pone.0050522.g003

Moreover, our models suggest that similar proportions of transmissions stem from undiagnosed, diagnosed but untreated, and inadequately-treated men in both countries. This argues that earlier treatment initiation and strategies to maintain retention in care and adherence to treatment must be linked to testing, and all should be included in comprehensive prevention packages. These data are consistent with recent findings from two different studies that $<30 \%$ of $\mathrm{HIV}$-infected Americans are virologically suppressed $[48,49]$.

None of the six CDC "evidence-based" interventions for MSM address transmission within stable partnerships, which comprise $35-39 \%$ of all infections in our results, comparable to other recent 


\section{Proportion of transmission by category - variation across single years: US, Model 2}

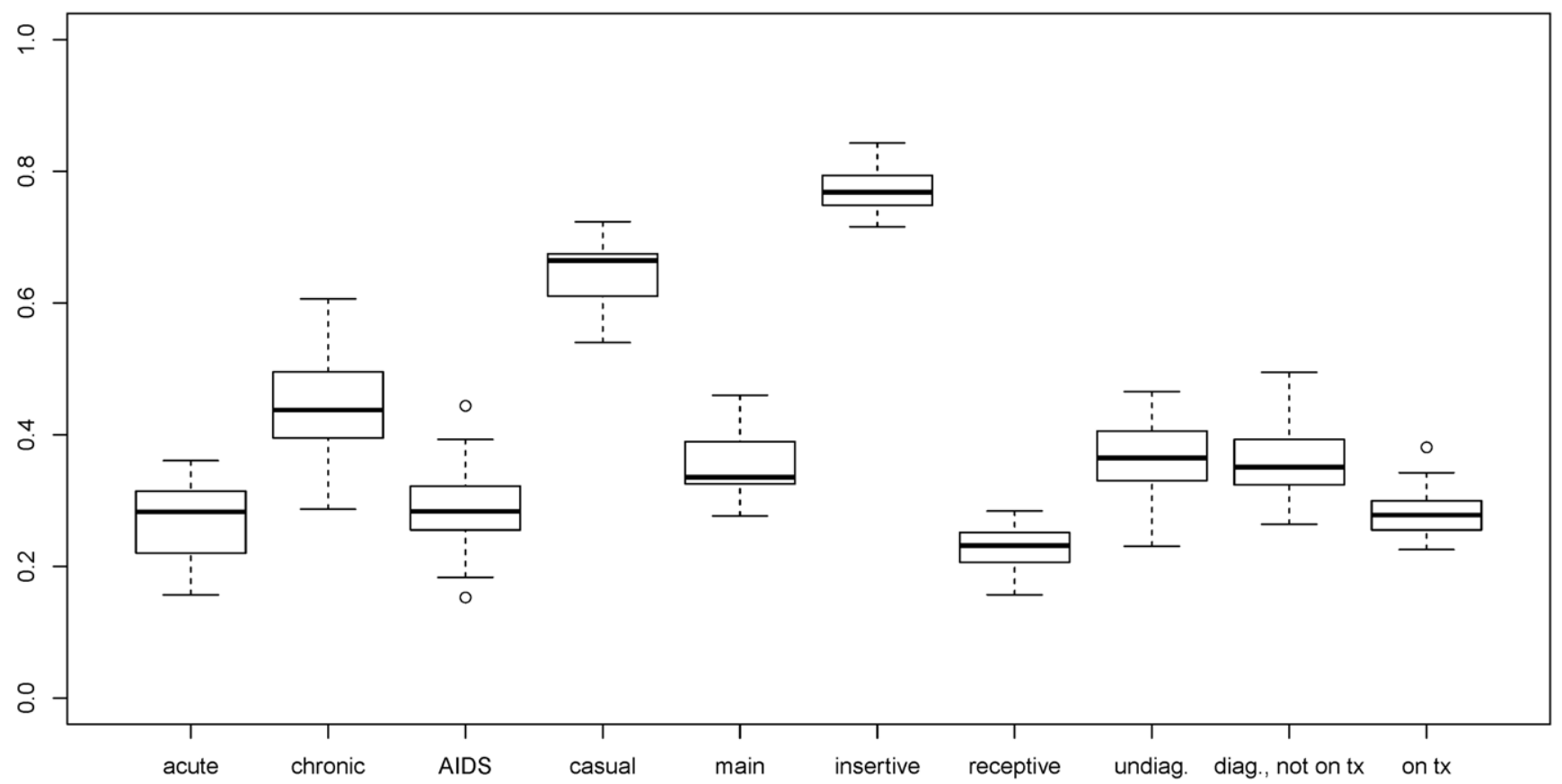

Figure 4. Sensitivity analyses. a) Changes in main partnership duration relative to base model (100\%). b) Changes in main and casual UAI relative to base model (100\%). c) Changes in testing frequency relative to base model (100\%). doi:10.1371/journal.pone.0050522.g004

estimates of $33 \%$ [11] and $26 \%$ [12]. While two recent developedworld estimates $(86 \%$ [14] and 68\% [13]) were considerably higher, the first of these defined stable partnerships more broadly and focused on young men, who switched steady partners frequently; the second is based on a simpler model that does not explicitly account for dynamic network structure, and uses a different estimate of relative UAI frequency in stable partnerships vs. casual contacts (Sullivan, personal communication). Despite the variability of these estimates, they are consistent in suggesting that substantial HIV transmission occurs in main partnerships in both the US and Peru, and thus support development and evaluation of behavioral interventions for MSM couples. Definitions for main partnerships were similar in source studies (generally reflecting emotional commitment), but the nature of such partnerships may differ considerably by country, and needs to be better understood.

Less is known about the proportion of HIV transmission from receptive to insertive anal sex partners. We estimated that $21 \%$ of transmissions are in this direction, similar to an earlier estimate of $28 \%$ [13], for US MSM; the slight difference may reflect the fact that our model explicitly requires the numbers of receptive and insertive acts to be equal. We found no estimates of transmissions by role for Peru. Our estimate of 33\% is higher than for the US, despite the apparent belief among some MSM in Lima that the insertive partner is at very low risk. This misconception leads to another: specifically, that exclusively insertive men are highly unlikely to be HIV infected and therefore pose no risk to their HIV negative receptive partners. Our findings suggest that messages addressing these misconceptions should figure prominently in risk reduction counseling for MSM with any sexual role, especially in the context of greater role exclusivity. Another implication is that promotion of circumcision might be a useful intervention among nearly or exclusively insertive MSM, at least in South America, where circumcision is relatively uncommon and seems protective among highly-insertive men [50]. However, cost and limited uptake could limit the effectiveness of this approach, especially since exclusively insertive men are disproportionately heterosexually-identified, and thus likely to not be open about their sexual activities with other men [51]. Counseling messages should emphasize that although receptive anal sex carries the highest risk, a substantial proportion of infections is also caused by insertive anal sex.

As with all models, our approach has numerous limitations. Some parameters are based on one data source, especially for Peru, and inputs are generally subject to bias and sampling error. Stage-specific estimates of per-act transmission were only available for heterosexuals; similar MSM measures are urgently needed. Although our model was more detailed than most, modeling always requires simplification, in part because data for more complicated models are commonly lacking. For example, we did not model temporal variability in treatment effectiveness, possibly leading to some underestimation of risk in main partnerships, where undetected treatment failure would be risky if partners relied on full suppression rather than condoms. Also, while our study represents an advance on earlier models in explicitly modeling main and casual partnerships separately, we did not explicitly model partnerships involving repeated contacts with partners not perceived as "main," but rather treated these as separate casual contacts. This could understate the importance of long-term relational concurrency, and provides no explicit guidance for counseling MSM about these relationships. It could also affect overall incidence in our model, and underestimate the proportion of infections in main partnerships by allowing someone to transmit to multiple casual partners in the model who are in reality the same person. In addition, we did not model contacts with females, a population at potentially substantial secondary risk in each setting. However, given the relatively low HIV prevalence 
among heterosexual women in both countries, female-to-male transmissions are unlikely to play any substantive risk for MSM in the Americas, as opposed to settings of generalized epidemics, e.g. southern Africa. We did not explicitly model male sex work or transgender identity, two aspects of MSM life of particular importance in Peru; however, men involved in either were included in source studies and thus contribute to model parameters. Finally, our data were limited to two countries-the US and Peru. Generalizing either model to other countries in the Americas should only be done with caution, as there remains much work to do in determining whether key inputs are similar for MSM in other settings.

In a pre-planned extension of this work, we will model the potential effects of various interventions in MSM, including preexposure prophylaxis (PrEP), which could dramatically change the prevention landscape for MSM. We will also assess the maximal predicted impact of voluntary medical male circumcision, which is likely to be incorporated into many prevention packages for heterosexuals in Africa, but whose impact in MSM, even in populations with substantial role segregation, is less clear. Using data from these models, we are building a combination prevention package for MSM in North and South America. The PUMA package is likely to include PrEP (with strategies to improve medication adherence), home HIV testing with online and telephone support and linkage to care, couples-based interventions, and more explicit risk reduction counseling information. We

\section{References}

1. Hall HI, Song R, Rhodes P, Prejean J, An Q, et al. (2008) Estimation of HIV incidence in the United States. JAMA 300: 520-529.

2. Beyrer G, Wirtz AL, Walker D, Johns B, Sifakis F, et al. (2011) The global HIV epidemics among men who have sex with men. Washington, DC: World Bank. p. p.

3. Prejean J, Song R, Hernandez A, Ziebell R, Green T, et al. (2011) Estimated HIV Incidence in the United States, 2006-2009. PLoS One 6: e17502.

4. Beyrer C, Baral SD, Walker D, Wirtz AL, Johns B, et al. (2010) The Expanding Epidemics of HIV Type 1 Among Men Who Have Sex With Men in Low- and Middle-Income Countries: Diversity and Consistency. Epidemiologic Reviews 32: 137-151.

5. Grant RM, Lama JR, Anderson PL, McMahan V, Liu AY, et al. (2010) Preexposure chemoprophylaxis for HIV prevention in men who have sex with men. N Engl J Med 363: 2587-2599.

6. Eshleman SH, Khaki L, Laeyendecker O, Piwowar-Manning E, Johnson-Lewis L, et al. (2009) Detection of individuals with acute HIV-1 infection using the ARCHITECT HIV Ag/Ab Combo assay. J Acquir Immune Defic Syndr 52: 121-124.

7. Cohen MS, Chen YQ, McCauley M, Gamble T, Hosseinipour MC, et al. (2011) Prevention of HIV-1 infection with early antiretroviral therapy. N Engl J Med 365: 493-505.

8. Gray RH, Kigozi G, Serwadda D, Makumbi F, Watya S, et al. (2007) Male circumcision for HIV prevention in men in Rakai, Uganda: a randomised trial. Lancet 369: 657-666.

9. Bailey RC, Moses S, Parker CB, Agot K, Maclean I, et al. (2007) Male circumcision for HIV prevention in young men in Kisumu, Kenya: a randomised controlled trial. Lancet 369: 643-656.

10. Auvert B, Taljaard D, Lagarde E, Sobngwi-Tambekou J, Sitta R, et al. (2005) Randomized, controlled intervention trial of male circumcision for reduction of HIV infection risk: the ANRS 1265 Trial. PLoS Med 2: e298.

11. Davidovich U, de Wit J, Albrecht N, Geskus R, Stroebe W, et al. (2001) Increase in the share of steady partners as a source of HIV infection: a 17-year study of seroconversion among gay men. AIDS 15: 1303-1308.

12. Jansen IAV, Geskus RB, Davidovich U, Jurriaans S, Coutinho RA, et al. (2011) Ongoing HIV-1 transmission among men who have sex with men in Amsterdam: a 25-year prospective cohort study. AIDS 25: 493-501.

13. Sullivan PS, Salazar L, Buchbinder S, Sanchez TH (2009) Estimating the proportion of HIV transmissions from main sex partners among men who have sex with men in five US cities. AIDS 23: 1153-1162.

14. Xiridou M, Geskus R, de Wit J, Coutinho R, Kretzschmar M (2003) The contribution of steady and casual partnerships to the incidence of HIV infection among homosexual men in Amsterdam. AIDS 17: 1029-1038.

15. Brenner BG, Roger M, Routy JP, Moisi D, Ntemgwa M, et al. (2007) High rates of forward transmission events after acute/early HIV-1 infection. J Infect Dis 195: 951-959 hope that through the combination of these prevention strategies for HIV uninfected persons and improved treatment coverage and adherence in HIV infected persons, we can halt the growing HIV epidemic in MSM throughout the Americas.

\section{Supporting Information}

\section{File S1 Online technical supplement. (DOCX)}

\section{Acknowledgments}

The authors would like to thank Al Liu, Jonathan Fuchs, Rivet Amico, Jen Sarche, Valdilea Veloso, Bill Snow, Matt Golden, Steven Wakefield, Ayn Leslie-Cook, Aditya Khanna, and Jerusha Achterberg; as well as the many data providers, including Kate Buchacz from HOPS and Alan Neaigus from NHBS (NYC). The views expressed herein do not necessarily reflect the official policies of the City and County of San Francisco; nor does mention of the San Francisco Department of Public Health imply its endorsement.

\section{Author Contributions}

Conceived and designed the experiments: SMG NBC EV JRLJS BG BAK KHM SPB. Performed the experiments: SMG NBC EV. Analyzed the data: SMG NBC EV JRL JS BG BAK KHM SPB. Contributed reagents/ materials/analysis tools: JRL JS BG KHM. Wrote the paper: SMG NBC EV SPB.

16. Brown AE, Gifford RJ, Clewley JP, Kucherer C, Masquelier B, et al. (2009) Phylogenetic reconstruction of transmission events from individuals with acute HIV infection: toward more-rigorous epidemiological definitions. J Infect Dis 199: 427-431

17. Lewis F, Hughes GJ, Rambaut A, Pozniak A, Leigh Brown AJ (2008) Episodic sexual transmission of HIV revealed by molecular phylodynamics. PLoS Med 5: e50.

18. Pao D, Fisher M, Hue S, Dean G, Murphy G, et al. (2005) Transmission of HIV-1 during primary infection: relationship to sexual risk and sexually transmitted infections. AIDS 19: 85-90.

19. Pinkerton SD (2007) How many sexually-acquired HIV infections in the USA are due to acute-phase HIV transmission? AIDS 21: 1625-1629.

20. Prabhu VS, Hutchinson AB, Farnham PG, Sansom SL (2009) Sexually acquired HIV infections in the United States due to acute-phase HIV transmission: an update. AIDS 23: 1792-1794.

21. Rapatski BL, Suppe F, Yorke JA (2005) HIV epidemics driven by late disease stage transmission. J Acquir Immune Defic Syndr 38: 241-253.

22. Wilson DP, Hoare A, Regan DG, Law MG (2009) Importance of promoting HIV testing for preventing secondary transmissions: modelling the Australian HIV epidemic among men who have sex with men. Sex Health 6: 19-33.

23. Xiridou M, Geskus R, de Wit J, Coutinho R, Kretzschmar M (2004) Primary HIV infection as source of HIV transmission within steady and casual partnerships among homosexual men. AIDS 18: 1311-1320.

24. Yerly S, Vora S, Rizzardi P, Chave JP, Vernazza PL, et al. (2001) Acute HIV infection: impact on the spread of HIV and transmission of drug resistance. AIDS 15: 2287-2292.

25. Marks G, Crepaz N, Janssen RS (2006) Estimating sexual transmission of HIV from persons aware and unaware that they are infected with the virus in the USA. AIDS 20: $1447-1450$.

26. (2010) Prevalence and awareness of HIV infection among men who have sex with men -21 cities, United States, 2008. MMWR Morb Mortal Wkly Rep 59: 1201-1207.

27. Sanchez J, Lama JR, Kusunoki L, Manrique H, Goicochea P, et al. (2007) HIV1 , sexually transmitted infections, and sexual behavior trends among men who have sex with men in Lima, Peru. Jaids-Journal of Acquired Immune Deficiency Syndromes 44: 578-585.

28. MacKellar DA, Gallagher KM, Finlayson T, Sanchez T, Lansky A, et al. (2007) Surveillance of HIV risk and prevention behaviors of men who have sex with men - A national application of venue-based, time-space sampling. Public Health Reports 122: 39-47.

29. Lama JR, Sanchez J, Suarez L, Caballero P, Laguna A, et al. (2006) Linking HIV and antiretroviral drug resistance surveillance in Peru: a model for a thirdgeneration HIV sentinel surveillance. J Acquir Immune Defic Syndr 42: 501505 .

30. Goicochea P, Sanchez J, Lucchetti A, Kusunoki L, Galvan R, et al. (2004) Successful recruitment and retention of high-risk men who have sex with men 
(MSM) in a preparedness cohort (HPTN 036) for an HSV-2 suppression trial: high observed incidence of HIV-1 infection. XV International AIDS Conference. Bangkok: International AIDS Society.

31. Celum C, Wald A, Hughes J, Sanchez J, Reid S, et al. (2008) Effect of aciclovir on HIV-1 acquisition in herpes simplex virus 2 seropositive women and men who have sex with men: a randomised, double-blind, placebo-controlled trial. Lancet 371: 2109-2119.

32. Chesney MA, Koblin BA, Barresi PJ, Husnik MJ, Celum CL, et al. (2003) An individually tailored intervention for HIV prevention: baseline data from the EXPLORE Study. Am J Public Health 93: 933-938.

33. Koblin BA, Chesney MA, Husnik MJ, Bozeman S, Celum CL, et al. (2003) High-risk behaviors among men who have sex with men in 6 US cities: baseline data from the EXPLORE Study. Am J Public Health 93: 926-932.

34. Handcock MS, Hunter DR, Butts CT, Goodreau SM, Morris M (2008) statnet: software tools for the statistical modeling of network data. 2.1 ed.

35. Wawer MJ, Gray RH, Sewankambo NK, Serwadda D, Li X, et al. (2005) Rates of HIV-1 transmission per coital act, by stage of HIV-1 infection, in Rakai, Uganda. J Infect Dis 191: 1403-1409.

36. Ma ZM, Stone M, Piatak M, Jr., Schweighardt B, Haigwood NL, et al. (2009) High specific infectivity of plasma virus from the pre-ramp-up and ramp-up stages of acute simian immunodeficiency virus infection. J Virol 83: 3288-3297.

37. Vaidya NK, Ribeiro RM, Miller CJ, Perelson AS (2010) Viral dynamics during primary simian immunodeficiency virus infection: effect of time-dependent virus infectivity. J Virol 84: 4302-4310.

38. Buchbinder SP, Katz MH, Hessol NA, O'Malley PM, Holmberg SD (1994) Long-term HIV-1 infection without immunologic progression. AIDS 8: 11231128 .

39. Little SJ, McLean AR, Spina CA, Richman DD, Havlir DV (1999) Viral dynamics of acute HIV-1 infection. J Exp Med 190: 841-850.

40. Wilson DP, Law MG, Grulich AE, Cooper DA, Kaldor JM (2008) Relation between HIV viral load and infectiousness: a model-based analysis (vol 372, pg 314, 2008). Lancet 372: 1808-1808.
41. Vittinghoff E, Douglas J, Judson F, McKirnan D, MacQueen K, et al. (1999) Per-contact risk of human immunodeficiency virus transmission between male sexual partners. Am J Epidemiol 150: 306-311.

42. Lyles RH, Munoz A, Yamashita TE, Bazmi H, Detels R, et al. (2000) Natural history of human immunodeficiency virus type 1 viremia after seroconversion and proximal to AIDS in a large cohort of homosexual men. Multicenter AIDS Cohort Study. J Infect Dis 181: 872-880.

43. Chu H, Gange SJ, Li X, Hoover DR, Liu C, et al. (2010) The effect of HAART on HIV RNA trajectory among treatment-naive men and women: a segmental Bernoulli/lognormal random effects model with left censoring. Epidemiology 21 Suppl 4: S25-34.

44. (2005) HIV prevalence, unrecognized infection, and HIV testing among men who have sex with men-five U.S. cities, June 2004-April 2005. MMWR Morb Mortal Wkly Rep 54: 597-601.

45. Ministry of Health of Peru (2006) A step forward in the fight against AIDS: the first two years of universal access to antiretroviral treatment in Peru. Lima: Ministry of Health.

46. Millett GA, Peterson JL, Wolitski RJ, Stall R (2006) Greater risk for HIV infection of black men who have sex with men: a critical literature review. Am J Public Health 96: 1007-1019.

47. Brenner BG, Roger M, Stephens D, Moisi D, Hardy I, et al. (2011) Transmission clustering drives the onward spread of the HIV epidemic among men who have sex with men in Quebec. J Infect Dis 204: 1115-1119.

48. (2011) Vital signs: HIV prevention through care and treatment-United States. MMWR Morb Mortal Wkly Rep 60: 1618-1623.

49. Gardner EM, McLees MP, Steiner JF, Del Rio C, Burman WJ (2011) The spectrum of engagement in HIV care and its relevance to test-and-treat strategies for prevention of HIV infection. Clin Infect Dis 52: 793-800.

50. Sanchez J, Sal YRVG, Hughes JP, Baeten JM, Fuchs J, et al. (2011) Male circumcision and risk of HIV acquisition among MSM. AIDS 25: 519-523.

51. Tabet S, Sanchez J, Lama J, Goicochea P, Campos P, et al. (2002) HIV, syphilis and heterosexual bridging among Peruvian men who have sex with men. AIDS 16: 1271-1277. 